\title{
Single-photon bus connecting spin-wave quantum memories
}

\author{
JONATHAN SIMON ${ }^{1,2 *}$, HARUKA TANJI1,2, SAIKAT GHOSH² AND VLADAN VULETIĆ2 \\ ${ }^{1}$ Department of Physics, Harvard University, Cambridge, Massachusetts 02138, USA \\ ${ }^{2}$ Department of Physics, MIT-Harvard Center for Ultracold Atoms, and Research Laboratory of Electronics, Massachusetts Institute of Technology, Cambridge, \\ Massachusetts 02139, USA \\ *e-mail: simonj@mit.edu
}

Generation of non-classical correlations (or entanglement) between atoms ${ }^{1-7}$, photons ${ }^{8}$ or combinations thereof ${ }^{9-11}$ is at the heart of quantum information science. Of particular interest are material systems serving as quantum memories that can be interconnected optically ${ }^{3,6,7,9-11}$. An ensemble of atoms can store a quantum state in the form of a magnon-which is a quantized collective spin excitation-that can be mapped onto a photon ${ }^{12-18}$ with high efficiency ${ }^{19}$. Here, we report the phasecoherent transfer of a single magnon from one atomic ensemble to another via an optical resonator serving as a quantum bus that in the ideal case is only virtually populated. Partial transfer deterministically creates an entangled state with one excitation jointly stored in the two ensembles. The entanglement is verified by mapping the magnons onto photons, whose correlations can be directly measured. These results should enable deterministic multipartite entanglement between atomic ensembles.

A quantum memory, that is, a device for storing and retrieving quantum states, is a key component of any quantum information processor. Optical memory access is highly desirable, as it is intrinsically fast and single photons are robust, easily controlled carriers of quantum states. Although a bit of quantum information (qubit) can be stored in a single two-level system, it can be expedient to instead use long-lived collective spin excitations of an atomic ensemble ${ }^{12}$. The ensemble can then be viewed as a 'macroatom', whose excitations are quantized spin waves (magnons), such that transitions between its energy levels (magnon number states) correspond to highly directional (superradiant ${ }^{20}$ ) photon emission or absorption $^{6,7,12-19}$.

Making use of the strong coupling between magnons and a single electromagnetic mode, single photons emitted by one sample have been captured in another ${ }^{15,16}$, demonstrating the single-photon character of the captured field ${ }^{15}$, but not phase coherence between the ensembles. Two ensembles can also be correlated by joint projective measurements ${ }^{3,6,14}$. This has been used to generate coherence between two macro-atoms within a single atomic cloud ${ }^{14}$, although entanglement between the ensembles was not verified ${ }^{21,22}$. For two remote atomic ensembles, a similar projective measurement has been used to generate probabilistic, but heralded ${ }^{23}$, entanglement ${ }^{6}$. Continuous spin variables of two atomic ensembles have also been entangled by joint measurement ${ }^{3}$.

Here, instead of using projective measurements of emitted fields for two-ensemble manipulation, we directly couple two macro-atoms, A and B, via an optical resonator. After probabilistic but heralded generation of a single magnon in macro-atom A, we transfer the magnon (or, if we choose, a portion of it) to macro-atom B while suppressing the population of the photonic mode by means of quantum interference (adiabatic dark-state transfer $)^{24-26}$. Successful transfer is verified by subsequent ondemand superradiant conversion of the magnon now stored in $\mathrm{B}$ into a photon. Partial transfer of the magnon creates a superposition state, where the two macro-atoms share a single spinwave quantum. This deterministically generates magnon-number entanglement between the two ensembles, as deduced by mapping the spin waves onto light fields, and carrying out quantumstate tomography.

Our set-up consists of two laser-cooled ensembles, A and B, of $N_{\mathrm{A}}, N_{\mathrm{B}} \sim 10^{3}$ caesium atoms each, inside a medium-finesse $(\mathcal{F}=240$ ) optical resonator (Fig. 1). The fundamental transverse mode of the resonator is weakly coupled to a single atom (singleatom cooperativity $\eta \approx 10^{-3} \ll 1$ ), but strongly to the magnon (collective cooperativity $N_{\mathrm{A}} \eta \sim N_{\mathrm{B}} \eta \sim 1$ ). When ensemble $\mathrm{A}$ is weakly illuminated with light, the detection (by a single-photon counting module) of a randomly emitted spontaneous Raman 'write' photon leaving the resonator heralds the creation of a quantized spin excitation ( spin Dicke state ${ }^{12,26}$ or magnon ${ }^{19}$ ) inside the ensemble. In the following, all quantities and states are conditioned on the detection of a write photon. For sufficiently low write probability, the ideal system is thereby prepared in the product state $|1\rangle_{A}|0\rangle_{B}$, specifying the number of magnons inside the corresponding macro-atom. By illuminating $A$ at a later time with a read pump $\Omega_{\mathrm{A}}$ that is phase-matched ${ }^{17}$ to the generated magnon and the optical-resonator mode, the magnon is converted into a read photon that can be detected on leaving the resonator (see the Supplementary Information). This mapping of collective spin excitations onto photons is key: all inferences about magnon states are derived from measured photon correlations (see the Methods section and the Supplementary Information).

If a pump beam $\Omega_{\mathrm{B}}$ is applied to ensemble $\mathrm{B}$ during readout of ensemble $A$, then the photon emitted by ensemble A into the resonator can be converted into a spin wave in ensemble B. In this case, the system is described by five mutually coupled collective states (Fig. 1c), where the outermost states, $\left|G_{\mathrm{A}}\right\rangle \equiv|1\rangle_{\mathrm{A}}|0\rangle_{\mathrm{B}}|0\rangle_{\mathrm{C}}$ and $\left|G_{\mathrm{B}}\right\rangle \equiv|0\rangle_{\mathrm{A}}|1\rangle_{\mathrm{B}}|0\rangle_{\mathrm{C}}$, correspond to a magnon stored in macro-atoms $A$ and $B$, respectively, with no photons in the cavity $(C) .\left|G_{\mathrm{A}}\right\rangle$ and $\left|G_{\mathrm{B}}\right\rangle$ are connected to each other through three intermediate states, $\left|E_{\mathrm{A}}\right\rangle \equiv|E\rangle_{\mathrm{A}}|0\rangle_{\mathrm{B}}|0\rangle_{\mathrm{C}}$, $\left|E_{\mathrm{B}}\right\rangle \equiv|0\rangle_{\mathrm{A}}|E\rangle_{\mathrm{B}}|0\rangle_{\mathrm{C}}$ and $|C\rangle \equiv|0\rangle_{\mathrm{A}}|0\rangle_{\mathrm{B}}|1\rangle_{\mathrm{C}}$, representing a collective 


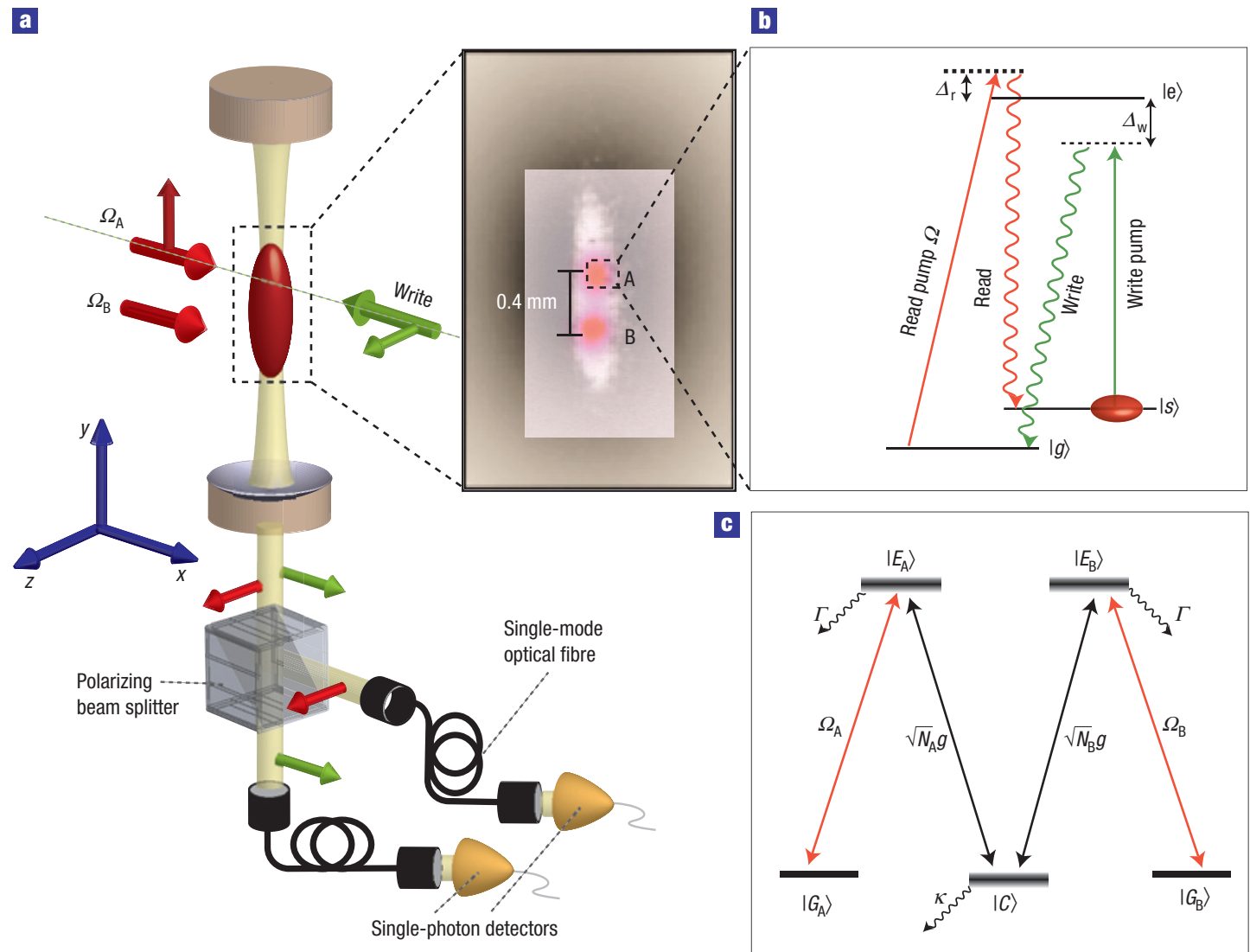

Figure 1 Set-up for phase-coherent optical transfer of a spin-wave quantum between two ensembles and entanglement generation. a, Simplified experimental set-up. Two ensembles, A and B, are defined within a cloud of laser-cooled caesium atoms by two read pump beams, $\Omega_{\mathrm{A}}$ and $\Omega_{\mathrm{B}}$. b. Heralded magnon generation. The collective spin excitation (magnon) is created within sample A by a weak spontaneous Raman process, $|s\rangle \rightarrow|e\rangle \rightarrow|g\rangle$, on application of a write pump beam on the transition $|S\rangle \rightarrow|e\rangle$, and heralded by the detection of a write photon on the $|e\rangle \rightarrow|g\rangle$ transition escaping from the resonator. c, Spin-wave transfer via adiabatic passage in a five-level system. The write process populates the state $\left|G_{A}\right\rangle \cdot \sqrt{N_{A}} g$ and $\sqrt{N_{B}} g$ are the collective couplings of the magnons to the optical resonator mode ${ }^{12}$, whereas $\Omega_{\mathrm{A}}$ and $\Omega_{\mathrm{B}}$ are pump couplings. In analogy to adiabatic passage in a three-level system ${ }^{27}$, application of a 'counter-intuitive pulse' sequence (see Fig. 2) transfers the system from $\left|G_{A}\right\rangle$ to $\left|G_{B}\right\rangle$, corresponding to magnon transfer from $A$ to $B$ via the optical resonator. Population of the photonic mode (state $|C\rangle$ ) and the corresponding excitation loss due to resonator decay are suppressed by quantum interference ${ }^{24,25}$. Re-absorption of the cavity photon by atoms within the cavity mode, but outside samples $A$ and $B$, is avoided by blue-detuning $\Omega_{\mathrm{A}}$ and $\Omega_{\mathrm{B}}$ from atomic resonance by $20 \mathrm{MHz}$.

electronic excitation in samples $\mathrm{A}$ and $\mathrm{B}$ and a photonic excitation in the cavity, respectively. Whereas $\left|G_{\mathrm{A}}\right\rangle$ and $\left|G_{\mathrm{B}}\right\rangle$ are long-lived, the intermediate states are unstable and decay via photon emission either into free space $\left(\left|E_{\mathrm{A}}\right\rangle\right.$ and $\left.\left|E_{\mathrm{B}}\right\rangle\right)$ or out of the resonator $(|C\rangle)$.

It may seem difficult to transport population through several short-lived intermediate states. However, as in the simpler case of a three-level system ${ }^{24-29}$, for sufficiently strong coupling the transfer $\left|G_{\mathrm{A}}\right\rangle \rightarrow\left|G_{\mathrm{B}}\right\rangle$ can be accomplished via adiabatic passage while suppressing the population of the intermediate unstable states by means of quantum interference. By applying a so-called counterintuitive pulse sequence, that is, by turning on pump $\Omega_{\mathrm{B}}$ coupling the initially empty level $\left|G_{\mathrm{B}}\right\rangle$ first, then ramping up pump beam $\Omega_{\mathrm{A}}$ and subsequently ramping down $\Omega_{\mathrm{B}}$, we are able to transfer the collective excitation from macro-atom A to macro-atom $B$ while reducing decay from $\left|E_{\mathrm{A}}\right\rangle,\left|E_{\mathrm{B}}\right\rangle$ or $|C\rangle$ (Fig. 2a). If the transfer was successful, the magnon can be directly converted into a photon by later application of pump $\Omega_{\mathrm{B}}$, which is automatically phase matched to the transferred magnon.

Figure $2 \mathrm{~d}$ shows the probability of detecting a read photon, conditioned on the previous detection of a write photon emitted from ensemble A. If we apply only pump $\Omega_{\mathrm{A}}$ at the transfer time,
$t_{\mathrm{T}}$, the A-magnon is converted into a photon with probability $R_{\mathrm{A}}=40 \%$ (Fig. 2e, blue dotted curve) and detected with an overall efficiency of $q_{\mathrm{e}}=0.11$ (2) due to losses and finite detector efficiency (see the Supplementary Information). As no collective excitations were written into sample $B$, no emitted photons are observed when pump $\Omega_{\mathrm{B}}$ is applied at a later time, $t_{\mathrm{B}}$ (see Fig. $2 \mathrm{f}$ ). However, if the adiabatic-transfer pulse sequence is applied at $t_{\mathrm{T}}$ (see Fig. 2b,c), then the collective excitation is transferred to sample $\mathrm{B}$, and converted into a photon when the pump beam $\Omega_{\mathrm{B}}$ is applied at $t_{\mathrm{B}}$. The application of $\Omega_{\mathrm{B}}$ also reduces the population of the cavity mode, $|C\rangle$, by destructive interference, as evident from the decrease in cavity emission during transfer at $t_{\mathrm{T}}$ in Fig. 2e. The transfer efficiency, $T_{\mathrm{AB}}$, from $\mathrm{A}$ to $\mathrm{B}$ is, depending on the optical depth (collective cooperativity) of the samples $N_{\mathrm{A}} \eta \simeq N_{\mathrm{B}} \eta \simeq 0.4-1$, between $T_{\mathrm{AB}}=10 \%$ and $25 \%$. A simple hamiltonian model provides good numerical agreement with this data, and predicts $T_{\mathrm{AB}}=\mathrm{e}^{\left(-\pi / \sqrt{N_{\mathrm{A}} \eta}\right)-\left(\pi / \sqrt{N_{\mathrm{B}} \eta}\right)}$ for large $N_{\mathrm{A}} \eta, N_{\mathrm{B}} \eta$.

As the magnon transfer process $\left|G_{\mathrm{A}}\right\rangle \rightarrow\left|G_{\mathrm{B}}\right\rangle$ corresponds to the net transfer of a photon from pump beam $\Omega_{\mathrm{A}}$ into pump beam $\Omega_{\mathrm{B}}$, the phase of the two-ensemble superposition state during the transfer is well defined and given by the phase difference $\phi$ between 

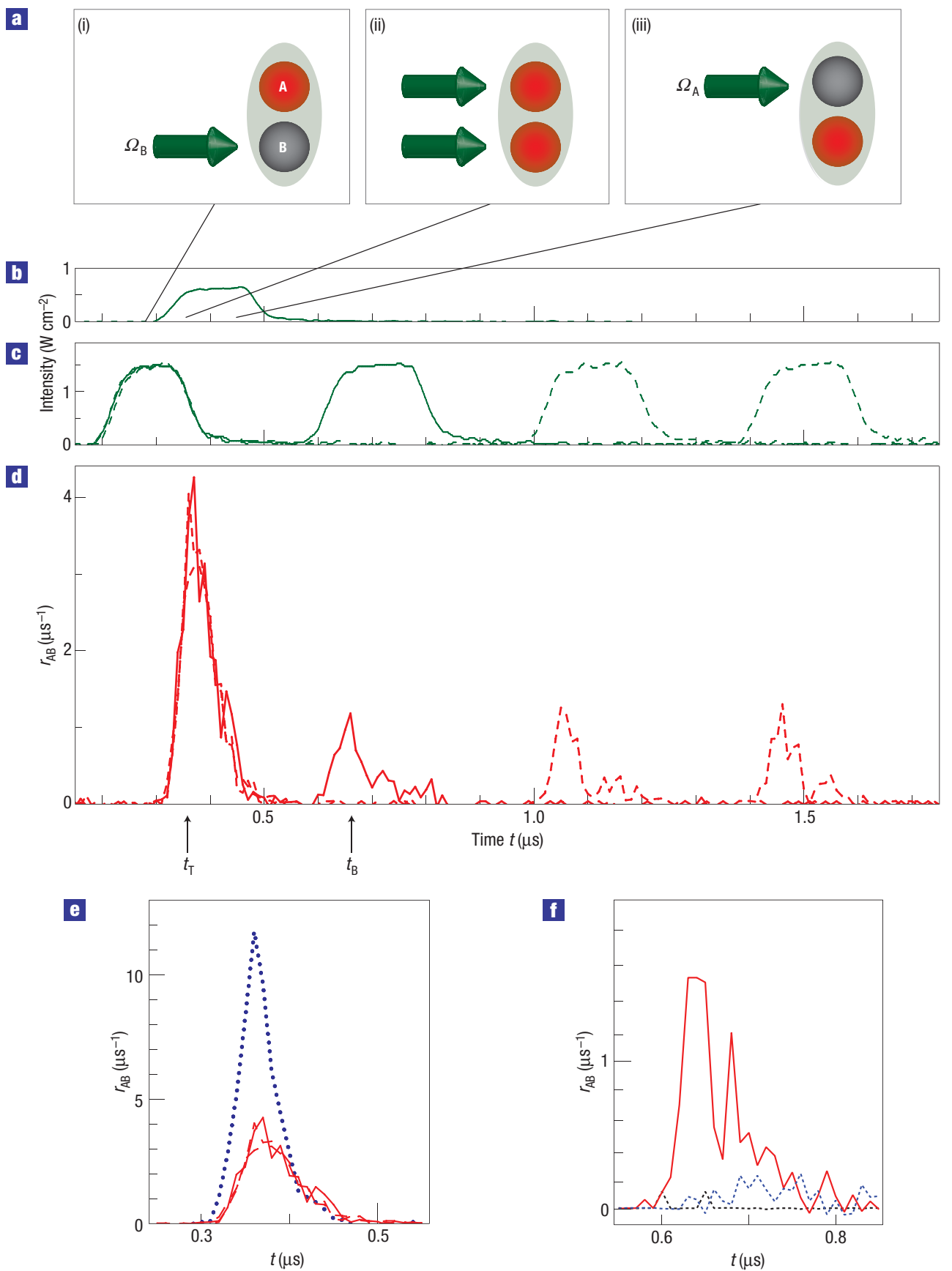

Figure 2 Magnon transfer from macro-atom A to macro-atom B. a, 'Counter-intuitive' pulse sequence. Following preparation of a magnon in sample A by the write process, the excitation is transferred according to the sequence: (i) pump beam $\Omega_{\mathrm{B}}$ is turned on, (ii) pump $\Omega_{\mathrm{A}}$ is ramped up and subsequently (iii) pump $\Omega_{\mathrm{B}}$ is ramped down - between (ii) and (iii) the magnon is shared between the two samples. When $\Omega_{\mathrm{B}}$ is fully extinguished (iii), the magnon has been transferred to ensemble $\mathrm{B}$. b,c, Applied pulse sequences of the pump beams $\Omega_{\mathrm{A}}(\mathbf{b})$ and $\Omega_{\mathrm{B}}$ (c). The dashed curves correspond to different read-out times from ensemble B. d, The read-photon probability density, conditioned on the preparation of a magnon in sample $\mathrm{A}, r_{\mathrm{AB}}$, as heralded by a detected write photon near $t=0$. The dashed curves show the read-out probability density from macro-atom B at various read times. e, Cavity leakage with (solid red curve) and without (dotted blue curve) magnon transfer to ensemble B. Incomplete suppression of the cavity leakage indicates imperfect adiabatic transfer. $\mathbf{f}$, Read-out of the B sample with (red solid curve) and without (blue dotted curve) the transfer process, demonstrating that the transfer was successful. The residual signal from B without transfer is a non-collective optical pumping signal resulting from a small overlap between sample B and the write beam applied to sample A.

$\Omega_{\mathrm{B}}$ and $\Omega_{\mathrm{A}}$. Consequently, if we interrupt the transfer process by advancing the turn-off time of $\Omega_{\mathrm{A}}$ until the magnon has some probability to be found in either macro-atom (adiabatic $\theta$ pulse), we expect, in the idealized limit of unit transfer efficiency and exactly one magnon in sample A before the transfer, to prepare the entangled (for $\theta \neq 0, \pi / 2$ ) state:

$$
|\theta, \phi\rangle=\cos \theta|1\rangle_{\mathrm{A}}|0\rangle_{\mathrm{B}}-\sin \theta \mathrm{e}^{i \phi}|0\rangle_{\mathrm{A}}|1\rangle_{\mathrm{B}},
$$


a

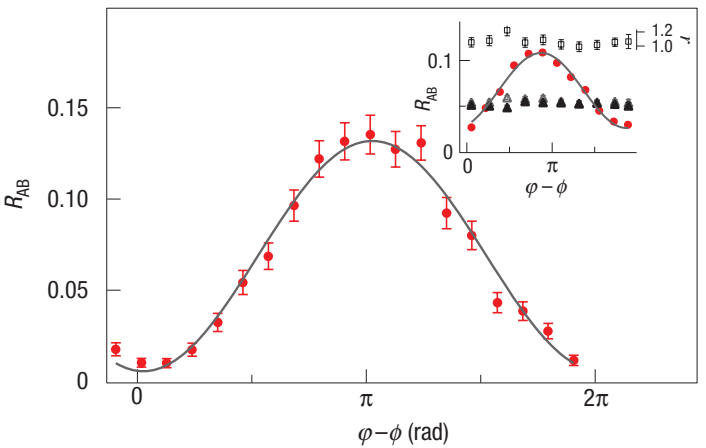

b

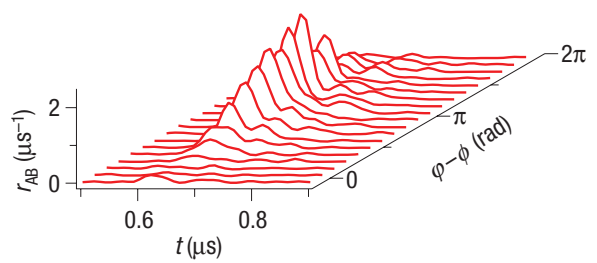

G

\begin{tabular}{|c|c|c|c|}
\hline$p_{00}$ & $p_{10}$ & $p_{01}$ & $p_{11}$ \\
\hline $0.88(1)$ & $0.08(1)$ & $0.030(4)$ & $0.0005(2)$ \\
\hline
\end{tabular}

Figure 3 Coherence of the two ensembles, A and B, after partial adiabatic transfer of a magnon. $\mathbf{a}$, By varying the phase difference $\varphi$ between the read pump beams during joint read-out of samples A and B for fixed preparation phase $\phi$, we measure the coherence between the atomic ensembles after a storage time of $200 \mathrm{~ns}$. The recovery efficiency, $R_{\mathrm{AB}}$ (red circles), is the read photon probability inside the resonator, conditioned on the previous detection of a write photon. Error bars indicate $\pm 1 \sigma$ intervals due to the finite number of counts. No backgrounds have been subtracted. The visibility is $V^{\text {photonic }}=0.88(4)$ for the measured ratio $r=p_{10} / p_{01}=2.54(5)$. The inset shows (for slightly different experimental conditions) that the individual read probabilities, $p_{10}, p_{01}$ (grey and black triangles, respectively) and $r$ (open squares), are independent of $\varphi$. $\mathbf{b}$, The joint read-out probability density referenced to within the cavity, conditioned on the preparation of a magnon in sample $\mathrm{A}$ at $t \simeq 0$. c, The measured probabilities $p_{i j}$ for $i$ and $j$ photons from samples $A$ and $B$, respectively, for separate read-out, referenced to within the cavity.

where the minus sign is characteristic of the dark state that leads to a suppression of population in the intermediate cavity state, $|C\rangle$ (see Fig. 1c). The state $|\theta, \phi\rangle$ can be viewed as an entangled state of the two ensembles or as a non-local qubit. The angles $\theta$ and $\phi$ can be adjusted by varying the relative turn-off time of, and the phase difference between, $\Omega_{\mathrm{A}}$ and $\Omega_{\mathrm{B}}$.

State (1) implies the existence of a well-defined phase (coherence) between its two components, $\left|G_{\mathrm{A}}\right\rangle=|1\rangle_{\mathrm{A}}|0\rangle_{\mathrm{B}}$ and $\left|G_{\mathrm{B}}\right\rangle=|0\rangle_{\mathrm{A}}|1\rangle_{\mathrm{B}}$. To measure this coherence, we apply, at a later time, both read pump beams $\Omega_{\mathrm{A}}$ and $\Omega_{\mathrm{B}}$ simultaneously, and vary the relative phase $\varphi$ of $\Omega_{\mathrm{B}}$ and $\Omega_{\mathrm{A}}$ during joint read-out. For $\varphi=\phi(\varphi=\phi+\pi)$, the read-out processes from the two macro-atoms interfere destructively (constructively), resulting in a sinusoidal dependence of the joint magnon-photon conversion efficiency on $\varphi-\phi$. Figure 3 shows the joint conversion versus $\varphi-\phi$ for a measured ratio $r=p_{10} / p_{01}=2.54(5)$, where $p_{i j}$ is the probability of detecting $i$ and $j$ photons for separate read-out of macro-atoms $\mathrm{A}$ and $\mathrm{B}$, respectively. Although we have also measured a fringe for other values of $r$, for example, $r=1.06(2)$ for the data shown in the inset, we have accumulated the largest data set and the best statistics for $r=2.54\left(\cos ^{2} \theta=0.71\right.$, or $\left.\theta=32^{\circ}\right)$. The measured fringe visibility of $V^{\text {photonic }}=0.88(4)$ is in good agreement with the maximum possible value of $V_{\max }^{\text {photonic }}=2 /\left(r^{1 / 2}+r^{-1 / 2}\right)=0.90(1)$. Incoherent double-excitation events could also potentially reduce the maximum possible fringe contrast; however, the reduction in our experiment falls within the statistical error.

Owing to imperfect single-magnon generation and magnon loss, our state also contains the two-magnon component $|1\rangle_{A}|1\rangle_{B}$ and the vacuum component $|0\rangle_{A}|0\rangle_{B}$, the combination of which may spoil the entanglement inherent in the ideal state (1). The system must then be described by a density matrix. As we can convert magnons from both samples individually or jointly into photons, we can carry out state tomography in the subspace $H_{1}=\{0,1\}_{\mathrm{A}} \otimes\{0,1\}_{\mathrm{B}}$ with zero or one excitations in each macroatom, and establish bounds on the density-matrix elements. From there it is possible to verify the presence of entanglement in the full system $^{6,23}$, for example, by establishing a bound on the concurrence $0 \leq \mathcal{C}_{\text {full }} \leq 1$ that measures the entanglement of formation ${ }^{30}$.

We adapt the procedure developed by Chou et al. ${ }^{6}$ for a similar system, where remote entanglement was generated by measurement. We denote the probability of finding $i$ and $j$ magnons in macro-atoms A and B, respectively, by $m_{i j}$, whereas the probability to find the system in the subspace $H_{1}$ is $M=m_{00}+m_{10}+m_{01}+m_{11}=1.00(1)$. The concurrence $\mathcal{C}_{\text {full }}$ can then be shown to obey $\mathcal{C}_{\text {full }} \geq \mathcal{C}$, where $\mathcal{C}$ is defined by

$$
\mathcal{C}=2 m\left(V-\sqrt{G_{\mathrm{AB}}}\right) .
$$

(See the Supplementary Information and ref. 6.) Here $m=\sqrt{m_{10} m_{01}}, G_{\mathrm{AB}}=m_{00} m_{11} /\left(m_{10} m_{01}\right)$ is closely related to the magnon-magnon cross-correlation function $g_{\mathrm{AB}}$ between the ensembles (and is bounded by it from above for $g_{A B} \leq 1$ ) and $V$ is the normalized magnon coherence within $H_{1}$ (see the Supplementary Information). Note that for poissonian magnon distributions in ensembles A and B we would have $G_{\mathrm{AB}}=1$ (see the Supplementary Information), whereas the coherence obeys by definition $V \leq 1$, and consequently $\mathcal{C} \leq 0$, so that there would be no entanglement. As the transfer process by itself cannot render subpoissonian magnon distributions in ensembles $\mathrm{A}$ and $\mathrm{B}$, entanglement requires the preparation of a single magnon in macro-atom A before transfer $\left(g_{\mathrm{AA}}<1\right)$. In a separate experiment, we have verified $g_{\mathrm{AA}} \leq g_{\mathrm{AA}}^{\text {photonic }}=0.13(8)<1$.

To evaluate equation (2), we note that an upper bound on $G_{\mathrm{AB}}$ is given by $g_{\mathrm{AB}}^{\text {photonic }}$ for the read photons after magnon-photon mapping (see the Methods section and the Supplementary Information). Similarly, a lower bound on $m=\sqrt{m_{10} m_{01}}$ is given by the detected read photon numbers referred back to within the resonator, and a lower bound on $V$ is given by $V \geq V^{\text {photonic }}$. From the measured values $V^{\text {photonic }}=0.88(4), g_{A B}^{\text {photonic }}=p_{11} /\left(p_{10}+p_{11}\right)\left(p_{01}+p_{11}\right)=0.21(8)$ and $m \geq 0.049(6)$ (see Fig. 3 ), we find $\mathcal{C} \geq 0.041(11)>0$ $(\mathcal{C} \geq 0.0046(11)>0$ without correcting for losses in the detection path), demonstrating that the two ensembles are entangled. $\mathcal{C}$ is primarily limited by the finite transfer and magnon-photon conversion efficiencies, or equivalently, by the value of, and bound we can place on, the vacuum component $|0\rangle_{\mathrm{A}}|0\rangle_{\mathrm{B}}$ compared with the ideal state (1). Both transfer and magnon-photon conversion ${ }^{19}$ can be significantly improved by increasing the optical depth of the system.

Our scheme is well suited to deterministic generation of entanglement over short distances. In the case of entanglement generation over lossy channels (that is, long distances), postselected schemes can be used to reduce the vacuum component and improve the available entanglement by repeated trials ${ }^{12}$. 
In summary, we have demonstrated a dark-state optical bus for phase-coherent transfer of a single spin-wave quantum between two atomic ensembles. It is noteworthy that entanglement can be deterministically generated in a linear system. Using optical dipole traps to enhance the optical depth of, and separation between, the samples would result in a concomitant increase in transfer efficiency, permitting entanglement of more than two macroatoms, and eventually multipartite entanglement on demand.

\section{METHODS}

The complete sequence consists of preparing cold caesium atoms in a magneto-optical trap, turning off the trapping fields and beams in $2 \mathrm{~ms}$, polarization-gradient cooling the atoms for $4 \mathrm{~ms}$, optically pumping for $100 \mu \mathrm{s}$ and then carrying out the write-transfer-read-out process repeatedly. The write-transfer-read-out process occurs once every $5 \mu$ s and is repeated 400 times, corresponding to $2 \mathrm{~ms}$ of data acquisition. Combined with the time required to recapture and re-cool the atoms, the entire sequence can be repeated at a rate of $15 \mathrm{~Hz}$

The two macro-atoms are each composed of approximately $10^{3}$ atoms within the cavity mode waist. The distance between the ensembles is $390 \mu \mathrm{m}$ and each sample has r.m.s. dimensions of $55 \mu \mathrm{m} \times 55 \mu \mathrm{m} \times 105 \mu \mathrm{m}$. The sample dimensions are defined in $\hat{x}$ and $\hat{y}$ by the cavity waist and in $\hat{z}$ by the beam waist.

The relevant atomic levels referenced in Fig. 1 are the hyperfine and magnetic sublevels $|s\rangle=\left|6 S_{1 / 2} ; F=4, m_{\mathrm{F}}=4\right\rangle,|e\rangle=\left|6 P_{3 / 2} ; 4,4\right\rangle$ and $|g\rangle=\left|6 S_{1 / 2} ; 3,3\right\rangle$.

The correlation functions for magnons are given by:

$$
g_{\mu \nu} \equiv \frac{\left\langle m_{\mu} m_{\nu}\right\rangle-\delta_{\mu \nu}\left\langle m_{\mu}\right\rangle}{\left\langle m_{\mu}\right\rangle\left\langle m_{\nu}\right\rangle},
$$

where $m_{\alpha}$ is the number of magnons in macro-atom $\alpha$ and $\delta_{\mu \nu}$ is the Kronecker delta function. The photon correlation functions are given by similar expressions.

Loss in the detection path does not affect the value of a (normally ordered) correlation function ${ }^{31}$. Throughout this work, we use that fact to bound the magnon correlation functions with their counterparts measured by converting the magnons into photons and measuring photon correlation functions. In addition to loss, the photonic paths have backgrounds that act to drive the correlation functions towards unity (or 2, in the case of thermal backgrounds). As such, magnon correlation functions that are less than unity $\left(g_{\mathrm{AA}}, g_{\mathrm{AB}}\right.$ and so on) are bounded from above by their photonic counterparts.

Our digital output board has a $15 \mathrm{~ns}$ timing resolution, resulting in a $10^{\circ}$ resolution for $\theta$, with a jitter of $4^{\circ}$ at $\theta=32^{\circ}$ resulting from atom number variation in the two samples, and pump laser detuning drift.

We operate at a write photon number per trial $n_{\mathrm{w}}=0.02(1)$ and find that the cross-correlation between the write photon and the read-out of the A-sample without transfer is $g_{\mathrm{WA}}=30(2)$. This is near the fundamental limit $g_{\mathrm{WA}}^{\max }=1 / n_{\mathrm{w}} \simeq 50$, indicating that the experiment is dominated by signal, and not by background.
Received 15 May 2007; accepted 20 August 2007; published 23 September 2007.

References

1. Turchette, Q. A. et al. Deterministic entanglement of two trapped ions. Phys. Rev. Lett. 81 , 3631-3634 (1998)

2. Rauschenbeutel, A. et al. Step-by-step engineered multiparticle entanglement. Science $\mathbf{2 8 8}$ 2024-2028 (2000)

3. Julsgaard, B., Kozhekin, A. \& Polzik, E. S. Experimental long-lived entanglement of two macroscopic objects. Nature 413, 400-403 (2001).

4. Leibfried, D. et al. Creation of a six-atom 'Schrödinger cat' state. Nature 438, 639-642 (2005).

5. Haffner, H. et al. Scalable multiparticle entanglement of trapped ions. Nature 438, 643-646 (2005).

6. Chou, C. W. et al. Measurement-induced entanglement for excitation stored in remote atomic ensembles. Nature 438, 828-832 (2005).

7. Matsukevich, D. N. et al. Entanglement of remote atomic qubits. Phys. Rev. Lett. 96, 030405 (2006),

8. Marcikic, I. et al. Distribution of time-bin entangled qubits over $50 \mathrm{~km}$ of optical fiber. Phys. Rev. Lett. 93, 180502 (2004).

9. Blinov, B. B., Moehring, D. L., Duan, L.-M. \& Monroe, C. Observation of entanglement between a single trapped atom and a single photon. Nature 428, 153-157 (2004).

10. Volz, J. et al. Observation of entanglement of a single photon with a trapped atom. Phys. Rev. Lett. 96, 030404 (2006)

11. Matsukevich, D. N. et al. Entanglement of a photon and a collective atomic excitation. Phys. Rev. Lett. 95, 040405 (2005).

12. Duan, L.-M., Lukin, M. D., Cirac, J. I. \& Zoller, P. Long-distance quantum communication with atomic ensembles and linear optics. Nature 414, 413-418 (2001).

13. Chou, C. W., Polyakov, S. V., Kuzmich, A. \& Kimble, H. J. Single-photon generation from stored excitation in an atomic ensemble. Phys. Rev. Lett. 92, 213601 (2004).

14. Matsukevich, D. N. \& Kuzmich, A. Quantum state transfer between matter and light. Science 306 663-666 (2004).

15. Chanelière, T. et al. Storage and retrieval of single photons transmitted between remote quantum memories. Nature 438, 833-836 (2005).

16. Eisaman, M. D. et al. Electromagnetically induced transparency with tunable single-photon pulses. Nature 438, 837-841 (2005).

17. Black, A. T., Thompson, J. K. \& Vuletić, V. On-demand superradiant conversion of atomic spin gratings into single photons with high efficiency. Phys. Rev. Lett. 95, 133601 (2005).

18. Thompson, J. K., Simon, J., Loh, H.-Q. \& Vuletić, V. A high-brightness source of narrowband, identical-photon pairs. Science 313, 74-77 (2006).

19. Simon, J., Tanji, H., Thompson, J. K. \& Vuletic, V. Interfacing collective atomic excitations and single photons. Phys. Rev. Lett. 98, 183601 (2007).

20. Dicke, R. H. Coherence in spontaneous radiation processes. Phys. Rev. 93, 99-110 (1954).

21. van Enk, S. J. \& Kimble, H. J. Comment on "Quantum state transfer between matter and light". Science 309, 1187b (2005).

22. Matsukevich, D. N. \& Kuzmich, A. Response to Comment on "Quantum state transfer between matter and light". Science 309, 1187c (2005).

23. van Enk, S. J., Lütkenhaus, N. \& Kimble, H. J. Experimental procedures for entanglement verification. Phys. Rev. A 75, 052318 (2007).

24. Parkins, A. S., Marte, P., Zoller, P. \& Kimble, H. J. Synthesis of arbitrary quantum states via adiabatic transfer of Zeeman coherence. Phys. Rev. Lett. 71, 3095-3098 (1993).

25. Pellizzari, T., Gardiner, S. A., Cirac, J. I. \& Zoller, P. Decoherence, continuous observation, and quantum computing: A cavity QED model. Phys. Rev. Lett. 75, 3788-3791 (1995).

26. Lukin, M. D., Yelin, S. F. \& Fleischhauer, M. Entanglement of atomic ensembles by trapping correlated photon states. Phys. Rev. Lett. 84, 4232-4235 (2000).

27. Bergmann, K., Theuer, H. \& Shore, B. W. Coherent population transfer among quantum states of atoms and molecules. Rev. Mod. Phys. 70, 1003-1025 (1998).

28. Kuhn, A., Hennrich, M. \& Rempe, G. Deterministic single-photon source for distributed quantum networking. Phys. Rev. Lett. 89, 067901 (2002).

29. McKeever, J. et al. Deterministic generation of single photons from one atom trapped in a cavity. Science 303, 1992-1994 (2004).

30. Wootters, W. K. Entanglement of formation of an arbitrary state of two qubits. Phys. Rev. Lett. 80, 2245-2248 (1998).

31. Mandel, L. \& Wolf, E. Optical Coherence and Quantum Optics (Cambridge Univ. Press, New York, 1995)

\section{Acknowledgements}

We gratefully acknowledge discussions with J. K. Thompson. This work is supported by the National Science Foundation and DARPA. J.S. acknowledges support from NDSEG and NSE.

Correspondence and requests for materials should be addressed to J.S.

Supplementary Information accompanies this paper on www.nature.com/naturephysics.

Reprints and permission information is available online at http://npg.nature.com/reprintsandpermissions/ 